\title{
Exercise, the Gut Microbiome, and Frailty
}

\author{
Hyung Eun Shin ${ }^{1}$, Seong Eun Kwak ${ }^{1}$, Ji-Hyun Lee ${ }^{1}$, Didi Zhang ${ }^{1}$, Jun Hyun Bae ${ }^{1}$, Wook Song ${ }^{1,2,3}$ \\ ${ }^{1}$ Health and Exercise Science Laboratory, Seoul National University, Seoul, Korea \\ ${ }^{2}$ Institute of Sport Science, Seoul National University, Seoul, Korea \\ ${ }^{3}$ Institue on Aging, Seoul National University, Seoul, Korea
}

Corresponding Author:

Wook Song, PhD

Institute of Sport Science, Seoul

National University, 1, Gwanak-ro,

Gwanak-gu, Seoul 08826, Korea

E-mail: songw3@snu.ac.kr

ORCID:

https://orcid.org/0000-0002-8825-6259

Received: March 26, 2019

Revised: June 13, 2019

Accepted: July 8, 2019
The gut microbiome is deeply associated with both skeletal muscle and brain function. In particular, gut microbiome dysbiosis may accelerate age-related diseases by affecting these systems. Although there is increasing evidence of the correlations between the gut microbiome and skeletal muscle and brain, it remains unclear whether changes in the gut microbiome due to exercise training can lead to healthy aging. This review covers the current status of gut microbiome-related research and future directions related to aging (e.g., physical frailty and cognitive dysfunction) as well as the effect of exercise training on both. We reviewed relevant literature including original articles and reviews identified from searches of the PubMed, Google Scholar, SCOPUS, EBSCOHost, ScienceDirect, Cochrane Library, and EMBASE databases using the following terms: 'gut microbiome', 'exercise', 'physical frailty', and 'cognitive dysfunction'. We identified a strong positive correlation between cognitive dysfunction or physical frailty and the gut microbiome. Furthermore, exercise had a significant effect on the composition of the gut microbiome. These results suggest that exercise training can prevent physical frailty or cognitive dysfunction by altering the gut microbiome. However, the exact mechanism by which these effects occur is not yet clear. Further studies are needed to determine whether exercise training can prevent age-related diseases by balancing the gut microbiome.

Key Words: Gastrointestinal microbiome, Frailty, Cognitive dysfunction, Exercise, Short chain fatty acids

\section{INTRODUCTION}

Previous studies have reported a direct link between disease and disability without adequately accounting for disability in the absence of disease. The concept of frailty was proposed to describe this condition. ${ }^{1)}$ Frailty is defined as a clinical state in which an individual is vulnerable to imbalanced homeostasis when exposed to a stressor event. ${ }^{2)}$ Frailty can occur as a consequence of a cumulative decline in a range of physiological conditions. ${ }^{3)}$ Although frailty is not a specific disease, it is a concept that encompasses clinical signs such as loss of weight, leisure time activity, gait speed, grip strength, and exhaustion. ${ }^{4)}$ Healthy aging refers to aging without such aging-related deficits. ${ }^{1)}$ It is obvious that exercise is a crucial tool to achieve healthy aging. The ultimate aim of exercise is to prevent aging-related deficits, leading to healthy aging. However, aging is also related to the gut microbiome. ${ }^{5)}$ It is becoming increasingly clear that the composition of the gut microbiome changes with age. Novel methods are being introduced that can lead to healthy aging through regulation of the composition of the gut microbiome. ${ }^{7)}$ Recent studies have shown that healthy centenarians have different gut microbiome characteristics compared to those of average older adults. ${ }^{8)}$ However, whether regulation of the gut microbiome through exercise training can lead to healthy aging is currently unclear. Therefore, in this review, we investigated whether alterations of the gut microbiome through exercise training can lead to healthy aging. The overview of study is shown in Fig. 1. 


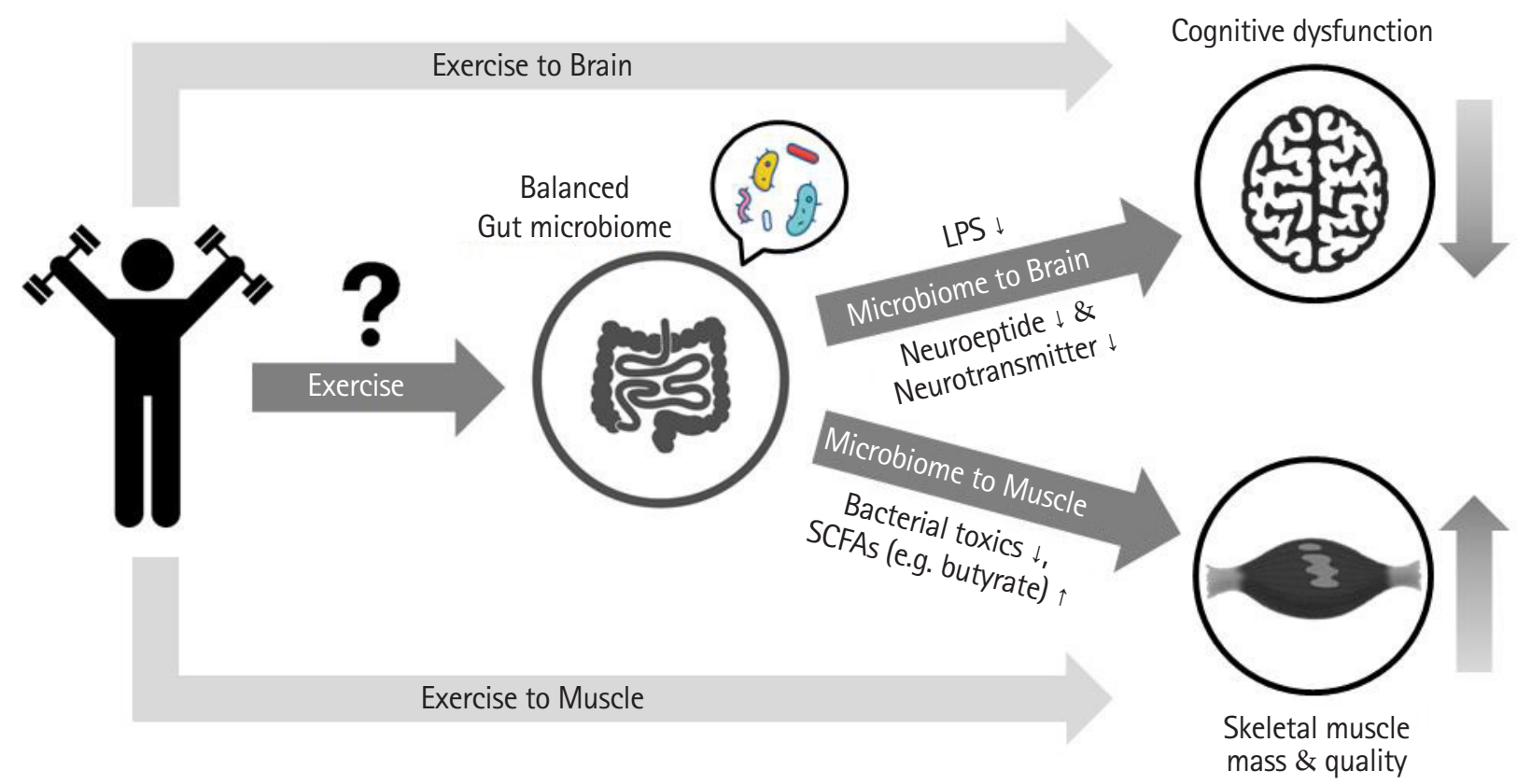

Fig. 1. Study overview: the gut-brain and gut-muscle axes. LPS, lipopolysaccharide; SCFAs, short-chain fatty acids.

\section{General Concepts of the Gut Microbiome}

The gut microbiome is an essential part of our body ${ }^{9)}$ It is involved in the regulation of various host metabolic pathways, leading to interactive host-microbiota metabolic signaling that connects the gut, muscle, and brain. ${ }^{10)}$ The host and its microbiota interact to produce gut microbiome-derived metabolites that contribute to the metabolic phenotype of the host. ${ }^{10)}$ The microbiome of the human gut includes at least 1,000 microbial species and approximately $10^{14}$ micro-organisms. The human microbiome is presumed to encode approximately $4 \times 10^{6}$ genes, approximately 150 times that in the human genome. ${ }^{11)}$ Recent studies have defined a host as a super-organism in which eukaryotic and prokaryotic cells co-exist. The gut microbiome is formed on the basis of the interaction of environmental factors, including lifestyle, the presence of disease, etc. ${ }^{12)}$ The microbiome may be altered by external and internal stimuli such as stress, diet, and antibiotic use. ${ }^{13)}$

Although the general characteristics of the gut microbiome in healthy people are not yet completely defined, the gut microbiomes of people with disease (e.g., metabolic syndrome, physical frailty, cognitive dysfunction, etc.) show a gradual change toward an imbalanced composition compared to those in healthy people. These imbalanced microbiome characteristics may contribute to disease onset and may play a role in a vicious cycle. ${ }^{14)}$ The gut microbiome coexists with the host in a symbiotic relationship and contributes to immune regulation and homeostasis. ${ }^{14)}$ Therefore, unbalanced gut microbiomes as a result of aging need to be pre- vented through exercise training and dietary habits. We investigated whether exercise training can balance the gut microbiome and contribute to healthy aging in the older population, with a focus on the relationship between the gut and muscle and the brain.

\section{Gut Microbiome and Exercise}

Exercise can significantly alter the composition of the gut microbiome, although the mechanism by which this occurs remains unclear. Some studies have assessed the effects of exercise as a treatment on metabolic disorders in mice with diabetes. When $d b / d b$ (type 2 diabetes [T2D]) and $d b /+$ (control) mice were made to exercise at a low intensity, the proportion of Bifidobacterium spp. increased in the $d b /+$ mice that exercised. ${ }^{9)}$ In another study, wildtype mice were subjected to voluntary wheel running for 12 weeks. After the exercise intervention, the Bacteroidetes:Firmicutes ratio increased, preventing diet-induced obesity. ${ }^{15)}$ In addition, 4-weekold C57BL/6J mice that were made to exercise on a treadmill had an increased relative abundance of Butyricimonas and Akkermansia. ${ }^{16)}$ The other effects of exercise on the gut microbiome composition of mice are summarized in Table 1.

It is obvious that exercise is associated with alterations in the composition of the gut microbiome. However, human studies have not investigated whether the gut microbiome is regulated by exercise. Allen et al. ${ }^{17)}$ showed that exercise can induce compositional and functional alterations in the human gut microbiome. Specifically, exercise increased the fecal concentration of short-chain fatty 
Table 1. Effects of exercise on the gut microbiome in mice

\begin{tabular}{|c|c|c|c|}
\hline Study & Model & Exercise & Outcomes \\
\hline Lambert et al. $\left.{ }^{9}\right)$ & $\begin{array}{l}d b / d b \text { (type } 2 \text { diabetes) and } d b /+ \\
(\text { control) mice }\end{array}$ & $\begin{array}{l}\text { Low-intensity treadmill } 5 \text { days/week for } 6 \\
\text { weeks }\end{array}$ & $\begin{array}{l}\uparrow \text { Firmicutes } \\
\downarrow \text { Bacteroides/Prevotella spp. in } d b / d b \text { and } d b /+ \text { ex- } \\
\text { ercised mice } \\
\uparrow \text { Bifidobacterium spp. in } d b /+ \text { exercised mice }\end{array}$ \\
\hline Mika et al. ${ }^{84)}$ & F344 rats (juvenile vs. adult) & Voluntary wheel running for 6 weeks & $\begin{array}{l}\uparrow \text { Bacteroidetes } \\
\downarrow \text { Firmicutes } \\
\uparrow \text { genera in juveniles }\end{array}$ \\
\hline Matsumoto et al. ${ }^{80)}$ & Wistar rats & Voluntary wheel running for 5 weeks & $\begin{array}{l}\uparrow \mathrm{n} \text {-butyrate concentration in exercised groups } \\
\uparrow \text { butyrate-producing bacteria }\end{array}$ \\
\hline Evans et al. ${ }^{15)}$ & Wild-type mice & Voluntary wheel running for 12 weeks & $\begin{array}{l}\uparrow \text { Bacteroidetes:Firmicutes ratio; prevent diet-in- } \\
\text { duced obesity mice }\end{array}$ \\
\hline Liu et al. ${ }^{13)}$ & 4-week-old C57BL/6J mice & Treadmill for 4 weeks & $\uparrow$ Butyricimonas and Akkermansia \\
\hline \multirow[t]{2}{*}{ Allen et al. ${ }^{81)}$} & 6-week-old C57BL/6J mice & Forced moderate treadmill running for 6 weeks & $\begin{array}{l}\downarrow \text { Turicibacter spp. in voluntary wheel running vs. } \\
\text { sedentary/forced treadmill running }\end{array}$ \\
\hline & & $\begin{array}{l}\text { Voluntary running wheels with free access for } \\
30 \text { days }\end{array}$ & \\
\hline Petriz et at. ${ }^{85)}$ & $\begin{array}{l}\text { About } 18 \text {-week-old obese Zucker rats, } \\
\text { hypertensive rats, Wistar rats }\end{array}$ & $30 \mathrm{~min} /$ day, 5 days/week for 4 weeks & $\begin{array}{l}\uparrow \text { Allobaculum (hypertensive rats) and Pseudomonas } \\
\text { and Lactobacillus (obese rats) were enriched after } \\
\text { exercise }\end{array}$ \\
\hline
\end{tabular}

acids (SCFAs) in lean participants. ${ }^{17)}$ Studies have also compared gut microbiomes between athletes and non-athletes. When compared with sedentary controls, Rugby Union players had increased levels of fecal metabolites such as SCFAs that are related to enhanced muscle turnover. ${ }^{18)}$ In addition, comparisons of the gut microbiomes of professional and amateur cyclists revealed a higher abundance of Methanobrevibacter smithii in professional cyclists. ${ }^{19)}$ Methanobrevibacter smithii upregulates genes associated with the production of methane by a metabolic pathway similar to that involved in the upregulation of energy and carbohydrate metabolism. ${ }^{19)}$ The other effects of exercise on the composition of the gut microbiome in humans are summarized in Table 2.

Table 2. Effects of exercise on the gut microbiomes in humans

\begin{tabular}{|c|c|c|c|}
\hline Study & Model & Exercise & Outcomes \\
\hline \multirow[t]{2}{*}{ Allen et al. ${ }^{17)}$} & $\begin{array}{l}20-45 \text { years of age, lean }(\mathrm{BMI}<25 \\
\left.\mathrm{kg} / \mathrm{m}^{2}\right) \text {, obese }\left(\mathrm{BMI}>30 \mathrm{~kg} / \mathrm{m}^{2}\right)\end{array}$ & $\begin{array}{l}\text { Supervised aerobic exercise ( } 3 \text { days/week for } 6 \\
\text { weeks) that progressed from } 30 \text { to } 60 \text { minutes/day } \\
\text { and from moderate ( } 60 \% \text { of HRR) to vigorous in- } \\
\text { tensity ( } 75 \% \text { HRR) }\end{array}$ & $\uparrow$ SCFAs in lean, but not in obese participants. \\
\hline & & & $\begin{array}{l}\text { Shifts in metabolic output of the microbiota paral- } \\
\text { leled alterations in SCFA-producing bacteria }\end{array}$ \\
\hline \multirow[t]{2}{*}{ Barton et al. ${ }^{18)}$} & Rugby Union players & Correlation (athletes vs. controls) & $\uparrow$ Fecal metabolites (SCFAs) in athletes \\
\hline & & & $\begin{array}{l}\uparrow \text { Amino acid, antibiotic biosynthesis, and carbo- } \\
\text { hydrate metabolism in athletes }\end{array}$ \\
\hline \multirow[t]{2}{*}{ Bressa et al. ${ }^{44)}$} & $\begin{array}{l}40 \text { premenopausal women } 18-40 \\
\text { years of age with BMI } 20-25 \mathrm{~kg} / \mathrm{m}^{2}\end{array}$ & $\begin{array}{l}\text { Sedentary women: } 3 \text { days of exercise/week for } 30 \\
\text { minutes }\end{array}$ & $\begin{array}{l}\uparrow \text { Faecalibacterium prausnitzii, Roseburia hominis, } \\
\text { and Akkermansia muciniphila in active women }\end{array}$ \\
\hline & & $\begin{array}{l}\text { Active women: } 3 \text { days of exercise/week for } 30 \text { min- } \\
\text { utes at a moderate intensity }\end{array}$ & \\
\hline Petersen et al. ${ }^{19)}$ & 33 cyclists & Professional vs. amateur cyclists & $\begin{array}{l}\uparrow \text { Abundance of Methanobrevibacter smithii in pro- } \\
\text { fessional cyclists (compared to amateur cyclists) }\end{array}$ \\
\hline
\end{tabular}

BMI, body mass index; HRR, heart rate reserve; SCFAs, short-chain fatty acids. 


\section{Gut Microbiome in Aging}

Age-related changes in the composition and diversity of the gut microbiome aggravate the immune system to regulate inflammatory responses. Collapse of the immune system causes age-related diseases. ${ }^{20)}$ The gut microbiome is related to the immune system in that both vary in composition with age. ${ }^{8)}$ Although the gut microbiota of humans is determined to some extent at birth, the composition continually changes throughout life according to the external environment. ${ }^{8,13)}$ This age-dependent gut microbiome is closely correlated with host inflammation and pathophysiology as the host ages. The gut physiology induced by this altered gut microbiome can cause host sensitivity to microbiota, leading to chronic and severe inflammatory responses.

Furthermore, these inflammatory responses can result in diseases such as cachexia, frailty, cancer, fatty liver disease, metabolic syndrome, T2D, and neurodegenerative diseases. ${ }^{20-22)}$ The gut microbiomes of older people are entirely different from those of younger adults. ${ }^{23)}$ With increasing age, the diversity of the gut microbiome decreases, with reduced numbers of Bifidobacteria, Firmicutes, Faecalibacterium prausnitzii, Clostridium cluster XIV, and Blautia coccoides-Eubacterium rectal. ${ }^{24)}$ However, inter-individual variations among individuals of the same age also exist because of various environmental factors. ${ }^{22)}$ In particular, the abundance of certain key species in older adults decreases, whereas the abundance of subdominant species increases. Also, the Firmicutes:Bacteroidetes ratio decreases in older individuals. ${ }^{25)}$ In one study, the gut microbiomes of older individuals exhibited a predominance of Bacteroidetes compared to those of younger individuals. ${ }^{26)}$ Bacteroidetes comprising Bacteroides, Alistipes, and Parabacteroides genera were the dominant core microbiota among older people. ${ }^{26)}$ These results clearly show that the gut microbiome changes with age. Therefore, we investigated how alterations in the gut microbiome could affect organs including the skeletal muscle and brain.

\section{Gut-Muscle Axis}

\section{Gut microbiome and physical frailty}

Physical frailty is common in the older population and is negatively correlated with health indicators. ${ }^{27)}$ Although the mechanism of physical frailty is not yet fully understood, the core components of physical frailty are strength, gait, body composition, and fatigue. Physical frailty, including weak muscle strength, slow gait speed, and poor balance, can lead to disability in performing activities of daily living. ${ }^{27)}$ Physical frailty is significantly associated with metabolic risk factors, independent of muscle loss. ${ }^{28)}$ Recent studies have clarified the relationship between physical frailty and the gut microbiome. Van Tongeren et al. ${ }^{29)}$ evaluated the relationship be- tween the gut microbiome diversity and frailty scores in the older population. Their results showed that the proportion of Lactobacilli, Bacteroides/Prevotella, and Faecalibacterium prausnitzii was significantly decreased, whereas the proportion of Enterobacteriaceae was significantly increased in older individuals with high frailty scores. ${ }^{29)}$ Claesson et al. ${ }^{6}$ also observed an association between the gut microbiome diversity and the Functional Independence Measure, the Barthel index (daily routine activity scale), and nutrition. Their results demonstrated a correlation between decreased gut microbiome diversity and increased frailty with high levels of inflammatory markers. ${ }^{6}$

Studies have shown that the gut microbiome is related to senescence. $^{20,21,30)}$ One study suggested that healthy gut microbiome characteristics, such as a high biodiversity, a high representation of SCFA producers, a representation of bacteria with beneficial metabolic activity, and a low representation of pathogens, are strongly correlated with a prolonged lifespan. ${ }^{31)}$ Healthy centenarians show remarkable gut microbiome characteristics compared with healthy older individuals. The gut microbiome of older adults who stay healthy is similar to that of young people aged $30-50$ years. ${ }^{32)}$ These studies are important because they indicate that positive changes in the gut microbiome through lifestyle habits may lead to improved healthy aging. In one study, individuals in a village with a high percentage of longevity were recruited to participate in gut microbiome research. The author studied eight centenarians (100-108 years of age), eight individuals aged 85-99 years residing in the same village, and eight older residents aged 80-92 years from other urbanized villages. Their results showed decreased ratios of Faecalibacterium and Akkermansia, increased ratios of Escherichia group and Methanobrevibacter, and altered ratios of Bacteroidetes in the gut microbiome of centenarians. ${ }^{33)}$ Another cohort study of individuals ranging from young children to semi-supercentenarians (105-109 years of age) investigated the overall changes in the gut microbiome profile with age. ${ }^{23)}$ The results indicated that the characteristics of the gut microbiome tended to significantly change with age. Notably, in adults over 95 years of age, the ratios of Faecalibacterium, Roseburia, Coprococcus, and Blautia were decreased, whereas those of Enterobacteriaceae were increased. $^{34)}$

To determine the changes in the gut microbiomes of semi-supercentenarians, Biagi et al..$^{8)}$ conducted a study including young adults (30 years old), older adults (65-75 years), centenarians (99-104 years), and semi-supercentenarians (105-109 years old). Their results demonstrated that the composition of the gut microbiome had a positive effect on the host's immune system; in that study, the proportions of Christensenellaceae, Akkermansia, and Bifidobacterium were significantly increased, although the gut microbiomes of 
centenarians and semi-supercentenarians had characteristics common to those of older populations. ${ }^{8)}$ More specifically, Akkermansia and Bifidobacterium are well-known health-related genera that can promote immune regulation, prevent inflammation, and maintain healthy metabolic homeostasis. ${ }^{8,35)}$ Future studies are needed to provide clear evidence that specific bacterial taxa can prolong life by maintaining metabolic homeostasis in older individuals.

Although age-related alterations in the gut microbiome have been studied extensively, the functional ability of the gut microbiome is not yet fully understood. One pilot study reported that the gut microbiome of centenarians showed increased proteolytic ability. ${ }^{7)}$ Although the abundance of genes related to carbohydrate metabolism markedly decreased, the abundance of genes associated with the metabolism of aromatic amino acids (e.g., tryptophan and phenylalanine) and other amino acids (lysine, valine) that are closely related to aging increased. ${ }^{7)}$ That pilot study demonstrated the need for more studies to confirm the potential functional capabilities of the gut microbiome.

\section{Gut microbiome and sarcopenia}

Frailty and sarcopenia are overlapping concepts that are common in older adults. ${ }^{36)}$ In particular, sarcopenia is a syndrome characterized by a lower muscle mass, quality, and strength. ${ }^{36)}$ Although there are numerous causes of sarcopenia, gut microbiota can affect skeletal muscle homeostasis through microbiota-induced metabolites, suggesting a possible biological basis toward the onset of sarcopenia. ${ }^{37)}$ Adequate control of the gut microbiome is required to prevent sarcopenia because the gut microbiome may be involved in the physiopathological mechanism of sarcopenia. The gut microbiome plays a crucial role in determining skeletal muscle mass, muscle structure, and muscle function. ${ }^{20,38)}$ Mice with sarcopenia have gut microbiome characteristics distinct from those of normal mice. $^{20,39)}$ SCFA producers, such as Faecalibacterium, Clostridium $X I V a$, and Butyricicoccus, are positively correlated with skeletal muscle mass. The microbiome can also contribute to muscle anabolism. ${ }^{39)}$ The circulation of SCFA byproducts of the gut microbiome, especially butyrate, in the body can positively influence skeletal muscle mass and function by regulating insulin sensitivity and inflammatory signals. ${ }^{40)}$ Furthermore, imbalances in the gut microbiome arise with age, which can cause a syndrome called 'leaky gut', in which gut microbiota can pass into the blood and promote inflammation. The activation of inflammation can inhibit the synthesis of skeletal muscle. ${ }^{40)}$ A previous study analyzing the serum microbiomes of younger (20-35 years) and older (60- 75 years) participants revealed a higher abundance of Bacteroidetes phylum than that in older participants. The increase in Bacteroidetes was positively related to the levels of insulin-like growth factor 1 (IGF-
1), which can act as an anabolic agent. ${ }^{41)}$ In contrast, inflammatory biomarkers such as interleukin 6 (IL-6) and tumor necrosis factor (TNF)-alpha are negatively correlated with the abundance of $\mathrm{Bac}$ teroidetes. $^{41)}$

\section{Effect of exercise on the gut-muscle axis}

As mentioned earlier, the proportions of bacteria that produce SCFAs are high in the gut microbiomes of centenarians. Older individuals with frailty show reduced representation of SCFA producers (e.g., Faecalibacterium prausnitzii). ${ }^{42)}$ As exercise training can increase the representation of SCFA producers, ${ }^{15)}$ alteration of gut microbiomes, especially SCFA producers, by exercise may improve physical frailty. Previous studies have shown that moderate-intensity exercise can lead to significant changes in the gut microbiome. For example, the representation of the Butyricimonas, Prevotella, and Akkermansia taxa increase. This can increase biodiversity and promote metabolic activity. ${ }^{16,43)}$ In addition, adult women with active lifestyles have higher rates of several health-promoting bacteria $(A k$ kermansia, Faecalibacterium, and Roseburia) than do age-matched women. ${ }^{44)}$ A recent study reported that athletes are more likely to express bacterial genes associated with SCFA-producing bacteria and carbohydrate amino acid metabolism compared with the general population. ${ }^{34)}$ This may contribute to higher concentrations of acetate, butyrate, and propionate in athletes. ${ }^{18)}$ These preliminary studies suggest that exercise training may prevent physical frailty by increasing SCFA producers in the gut microbiome.

Among the byproducts of the gut microbiome, SCFAs have been the most studied. They mainly act on skeletal muscle and mitochondria by promoting insulin sensitivity, inflammation regulation, and anabolism. ${ }^{45,46)}$ SCFAs produced by the gut microbiome (e.g., Faecalibacterium, Butyricimonas, etc.) can enter systemic circulation and be absorbed into skeletal muscle. Free fatty acid receptors 2 and 3 (FFAR-2 and FFAR-3) can promote insulin sensitivity and regulate glucose uptake. ${ }^{47)}$ SCFAs can also activate mitochondrial biosynthesis regulator NAD-dependent deacetylase sirtuin-1 (SIRT1) receptors. ${ }^{48)}$ The most intriguing mediator among SCFA is butyrate, which affects the activity of several regulatory pathways (e.g., UCP2-AMPK-ACC and PGC1-alpha) and improves ATP production and myofiber metabolism efficiency. ${ }^{49)}$ Treatment with probiotics, including the major SCFA producer Faecalibacterium prausnit$z i i$, was also effective in reducing systemic inflammation in mice and promoting assimilation to produce healthy muscles. ${ }^{50)}$

\section{Gut-Brain Axis}

Gut microbiome and cognitive dysfunction

Recently, the concept of frailty has been focused mainly on physical frailty. ${ }^{51)}$ However, studies on cognitive frailty have also begun 
to attract attention. ${ }^{52)}$ Cognitive frailty is defined as a syndrome in older individuals with physical frailty and cognitive dysfunction. ${ }^{53)}$ Cognitive dysfunction can cause neurodegenerative disorders such as Alzheimer disease (AD) and Parkinson disease. ${ }^{27,54)}$ Recent studies have reported a strong association between imbalance in the gut microbiome and cognitive dysfunction (e.g., dementia). ${ }^{11,55,56)}$ In particular, gut microbiomes known to be harmful to the host can accelerate the onset of dementia. ${ }^{57)}$ Studies have focused on microbial byproducts in blood or brain tissue to investigate the potential role of the gut microbiome in the development of dementia. ${ }^{56)}$ The percentages of mannitol, succinic acid, and 3,4-dihyroxy benzeneacetic acid, which are byproducts of microorganisms, are higher in patients with $\mathrm{AD}$, who have different gut micro-organisms compared to those in normal controls. ${ }^{58)}$ In addition, lipopolysaccharide (LPS) derived from Gram-negative bacteria has been recently reported in the hippocampal and neocortex tissues of patients with $\mathrm{AD}^{59)}$ An imbalance of the gut microbiome in these patients can cause the accumulation of by-products in brain tissue. ${ }^{56)}$ The amount of Clostridium difficile in patients with dementia was significantly higher than that in patients without dementia. ${ }^{60)}$ According to the $16 \mathrm{~S}$ rRNA microbial profile, the gut microbiome diversity is significantly lower in people with Clostridium clustering. ${ }^{60,61)}$ Cyanobacteria within the gut microbiota can synthesize neurotoxins such as saxitoxin and alpha-anatoxin. ${ }^{62)}$ Moreover, Citrobacter, Escherichia coli, Klebsiella, Mycobacteria, Pseudomonas, Streptococcus, Streptomyces, Staphylococcus, Salmonella, and Bacillus spp. in the gut microbiota can synthesize amyloid peptides. ${ }^{62,63)}$ These peptides can be transmitted and accumulate in the brain, resulting in cognitive dysfunction or dementia. ${ }^{64)}$ Also, reduction of Bifidobacteria and Eubacterium rectale is associated with biomarkers of $\mathrm{AD}{ }^{55)}$ These results suggest that the gut microbiome may be an essential factor in the pathogenesis of dementia, although the apparent causal relationship between the gut microbiome and neurodegeneration has not yet been elucidated. ${ }^{65)}$ In one study, comparison of the gut microbiomes of amyloid-positive patients, amyloid-negative patients, and control individuals revealed a low ratio of Eubacterium rectale and a high proportion of Escherichia/Shigella in amyloid-positive patients. ${ }^{52)}$ Differences in gut microbiomes play a role in controlling amyloid accumulation in the brain through immune regulation. ${ }^{66)}$ Although the gut microbiome and dementia are highly related, there is limited research on the association of mild cognitive impairment (MCI) with the gut microbiome. Additionally, the relative proportions of microorganisms of the gut microbiota, such as Bifidobacterium, Butyricicoccus, and Clostridium XIVb, were negatively correlated with the presence of cognitive dysfunction in patients with Parkinson disease. $^{67)}$
Effect of exercise on gut-brain axis

Although studies have elucidated a relationship between gut health and the brain, ${ }^{11,56)}$ the mechanisms by which the exercise-induced gut environment can influence cognitive function remain unknown. Exercise may have a significant effect on cognitive function by altering the gut microbiome because of its strong relationship with cognitive function; thus, exercise may result in positive changes in the gut environment.

The gut-brain axis is a bidirectional communication channel that is regulated by hormones, immunity, and nerve signals. A wellknown characteristic of SCFAs is the prevention of obesity by increasing the expressions of glucagon-like peptide 1 and peptide YY, which can induce satiety. ${ }^{68,69)}$ In addition, sodium butyrate treatment is effective in increasing the expression of brain-derived neurotrophic factor (BDNF), which acts as an antidepressant. ${ }^{70)}$ Taken together, an increase in the number of SCFA-producing bacteria resulting from exercise seems to have a potentially significant and beneficial effect on the gut-brain axis.

Exercise affects the gut-brain axis by controlling vagus nerve tension. ${ }^{71)}$ The vagus nerve affects anti-inflammatory immune regulation and the imbalance of modified vagal activity. The hypothalamic-pituitary-adrenal (HPA) axis is typically affected in patients with depression and inflammatory bowel disease (IBD). ${ }^{72,73)} \mathrm{Al}-$ though the potential effects of extrinsic vagal nerve stimulation (VNS) on neuroimmunomodulation are poorly studied, VNS may be effective in controlling conditions such as depression, IBD, etc. that are difficult to treat. ${ }^{73)}$ The effect of the vagus nerve on the gut microbiome during exercise requires study. ${ }^{73)}$ However, steady aerobic exercise can lead to a decrease in the resting heart rate and an increase in the input of the vagus nerve to the sinus node. Increased parasympathetic nerve stimulation can last for a long time. Regular aerobic exercise can have the same effect as VNS to induce a potential rise in the cholinergic anti-inflammatory pathway. ${ }^{74,75)}$ Although research is lacking, exercise-induced activation of the vagus nerve in the gut microbiome may prevent brain disease.

\section{DISCUSSION}

With the gradual development of social and economic environments, sedentary lifestyles are associated with metabolic syndromes, such as obesity, diabetes, etc., that can promote aging. ${ }^{76)}$ These imbalances can be improved through exercise training to maintain homeostasis. Many mechanisms are involved in the beneficial effects of exercise training on health. Exercise training can activate anti-inflammatory responses, promote the HPA axis, and enforce neuromuscular function. ${ }^{77,78)}$ Recently, physical activity has been proposed to alter gut micro-organisms. In addition, exer- 
cise can promote a healthy state by improving the gut microbiome. However, little is known about the effect of increased physical activity through exercise training on the gut microbiome. Exercise training may lead to positive changes in the gut microbiome. However, the direct or indirect mechanisms by which exercise training does so remain uncertain. It is difficult to elucidate the long-term effects of exercise because the gut microbiota is influenced by several genetic and environmental factors. ${ }^{79)}$ For this reason, previous studies have primarily sought to demonstrate the correlation between the gut microbiome and physical function. These studies have shown that the gut microbiome has distinct characteristics. ${ }^{9,16,80)}$ This led to the hypothesis that improvements in physical function through exercise training could also be associated with the gut microbiome. On the basis of the effects of exercise that addressed in this review paper, exercise may be a feasible method for preventing or delaying aging.

With age, the gut microbiome becomes imbalanced, which can lead to age-related diseases such as physical frailty and cognitive dysfunction. These geriatric diseases can be effectively controlled by exercise. We examined whether the changes to the gut microbiome resulting from exercise training could have a positive effect on these diseases. Previous studies have reported changes in the diversity of the gut microbiome and specific bacterial groups with exercise training. ${ }^{9,16,80,81)}$ Therefore, it is important to study alterations in the gut microbiome according to the type and intensity of exercise. However, to our knowledge, no studies have determined which exercise types (e.g., resistance or aerobic exercise) are more effective in influencing the gut microbiome. In previous animal studies, alterations in the gut microbiome through exercise training have been studied mainly in aerobic exercise such as a voluntary running wheel, treadmill, etc. because of the limitations of resistance training. ${ }^{80,82)}$ Previous studies categorized aerobic exercise as low-, medium-, and high-intensity to investigate changes in the gut microbiome. ${ }^{9,16,83)}$ However, direct comparison of exercise intensity to determine the most appropriate intensity has not yet been reported. Thus, studies are required to determine the most appropriate exercise intensity to prevent age-related brain and metabolic diseases through the gut microbiome.

\section{CONCLUSION}

By interacting with the host, the gut microbiome has an enormous impact on the entire body. Recent studies have consistently reported that the gut microbiome is related to geriatric diseases. As the gut microbiome is changed in the older individuals with altered physiology, it is necessary to determine whether the gut microbiome is involved in the improvement of physical function by exer- cise and whether exercise training can prevent geriatric diseases in future studies.

\section{CONFLICT OF INTEREST DISCLOSURES}

The researchers claim no conflicts of interest.

\section{ACKNOWLEDGEMENTS}

This work was supported by grants of Korea Mouse Phenotyping Project (No. NRF-2013M3A9D5072550, No. 2013M3A9D 5072560, No. 2017M3A9D5A01052447, and No. MEST-2011030135) from the National Research Foundation of Korea funded by the Ministry of Science, ICT and Future Planning (No. NRF2013M3A9B6046417).

\section{REFERENCES}

1. Garcia-Garcia FJ, Gutierrez Avila G, Alfaro-Acha A, Amor Andres MS; De Los Angeles De La Torre Lanza M, Escribano Aparicio $\mathrm{MV}$, et al. The prevalence of frailty syndrome in an older population from Spain. The Toledo Study for Healthy Aging. J Nutr Health Aging 2011; 15:852-6.

2. MorleyJE, Vellas B, van Kan GA, Anker SD, Bauer JM, Bernabei $\mathrm{R}$, et al. Frailty consensus: a call to action. J Am Med Dir Assoc 2013;14:392-7.

3. Clegg A, Young J, Iliffe S, Rikkert MO, Rockwood K. Frailty in elderly people. Lancet 2013;381:752-62.

4. Rodriguez-Manas L, Feart C, Mann G, Vina J, Chatterji S, Chodzko-Zajko W, et al. Searching for an operational definition of frailty: a Delphi method based consensus statement: the frailty operative definition-consensus conference project. J Gerontol A Biol Sci Med Sci 2013;68:62-7.

5. Choi YJ, Lee DH. Microbiota: A Key for Healthy Aging. Ann Geriatr Med Res 2016;20(4):168-76.

6. Claesson MJ, Jeffery IB, Conde S, Power SE, O’Connor EM, Cusack S, et al. Gut microbiota composition correlates with diet and health in the elderly. Nature 2012;488:178-84.

7. Rampelli S, Candela M, Turroni S, Biagi E, Collino S, Franceschi $\mathrm{C}$, et al. Functional metagenomic profiling of intestinal microbiome in extreme ageing. Aging (Albany NY) 2013;5:902-12.

8. Biagi E, Franceschi C, Rampelli S, Severgnini M, Ostan R, Turroni S, et al. Gut microbiota and extreme longevity. Curr Biol 2016;26:1480-5.

9. Lambert JE, Myslicki JP, Bomhof MR, Belke DD, Shearer J, Reimer RA. Exercise training modifies gut microbiota in normal and diabetic mice. Appl Physiol Nutr Metab 2015;40:749-52. 
10. Nicholson JK, Holmes E, Kinross J, Burcelin R, Gibson G, Jia W, et al. Host-gut microbiota metabolic interactions. Scienc 2012; 336:1262-7.

11. Jiang C, Li G, Huang P, Liu Z, Zhao B. The gut microbiota and Alzheimer's disease. J Alzheimers Dis 2017;58:1-15.

12. Cho I, Blaser MJ. The human microbiome: at the interface of health and disease. Nat Rev Genet 2012;13:260-70.

13. Bokulich NA, Chung J, Battaglia T, Henderson N, Jay M, Li H, et al. Antibiotics, birth mode, and diet shape microbiome maturation during early life. Sci Transl Med 2016;8:343ra82.

14. Romani-Perez M, Agusti A, Sanz Y. Innovation in microbiome-based strategies for promoting metabolic health. Curr Opin Clin Nutr Metab Care 2017;20:484-91.

15. Evans CC, LePard KJ, Kwak JW, Stancukas MC, Laskowski S, Dougherty J, et al. Exercise prevents weight gain and alters the gut microbiota in a mouse model of high fat diet-induced obesity. PLoS One 2014;9:e92193.

16. Liu Z, Liu HY, Zhou H, Zhan Q, Lai W, Zeng Q, et al. Moderate-intensity exercise affects gut microbiome composition and influences cardiac function in myocardial infarction mice. Front Microbiol 2017;8:1687.

17. Allen JM, Mailing LJ, Niemiro GM, Moore R, Cook MD, White $\mathrm{BA}$, et al. Exercise alters gut microbiota composition and function in lean and obese humans. Med Sci Sports Exerc 2018; 50:747-57.

18. Barton W, Penney NC, Cronin O, Garcia-Perez I, Molloy MG, Holmes E, et al. The microbiome of professional athletes differs from that of more sedentary subjects in composition and particularly at the functional metabolic level. Gut 2018;67:625-33.

19. Petersen LM, Bautista EJ, Nguyen H, Hanson BM, Chen L, Lek $\mathrm{SH}$, et al. Community characteristics of the gut microbiomes of competitive cyclists. Microbiome 2017;5:98.

20. Ticinesi A, Tana C, Nouvenne A. The intestinal microbiome and its relevance for functionality in older persons. Curr Opin Clin Nutr Metab Care 2019;22:4-12.

21. Ticinesi A, Lauretani F, Milani C, Nouvenne A, Tana C, Del Rio $\mathrm{D}$, et al. Aging gut microbiota at the cross-road between nutrition, physical frailty, and sarcopenia: is there a gut-muscle axis? Nutrients 2017;9:1303.

22. Vaiserman AM, Koliada AK, Marotta F. Gut microbiota: a player in aging and a target for anti-aging intervention. Ageing Res Rev 2017;35:36-45.

23. O'Toole PW, Jeffery IB. Gut microbiota and aging. Science 2015;350:1214-5.

24. Rondanelli M, Giacosa A, Faliva MA, Perna S, Allieri F, Castellazzi AM. Review on microbiota and effectiveness of probiotics use in older. World J Clin Cases 2015;3:156-62.
25. Perez Martinez G, Bauerl C, Collado MC. Understanding gut microbiota in elderly's health will enable intervention through probiotics. Benef Microbes 2014;5:235-46.

26. Claesson MJ, Cusack S, O’Sullivan O, Greene-Diniz R, de Weerd $\mathrm{H}$, Flannery E, et al. Composition, variability, and temporal stability of the intestinal microbiota of the elderly. Proc Natl Acad Sci U S A 2011;108 Suppl 1:4586-91.

27. Buchman AS, Schneider JA, Leurgans S, Bennett DA. Physical frailty in older persons is associated with Alzheimer disease pathology. Neurology 2008;71:499-504.

28. Lee JS, Auyeung TW, Leung J, Kwok T, Leung PC, Woo J. Physical frailty in older adults is associated with metabolic and atherosclerotic risk factors and cognitive impairment independent of muscle mass. J Nutr Health Aging 2011; 15:857-62.

29. van Tongeren SP, Slaets JP, Harmsen HJ, Welling GW. Fecal microbiota composition and frailty. Appl Environ Microbiol 2005;71:6438-42.

30. Debebe T, Biagi E, Soverini M, Holtze S, Hildebrandt TB, Birkemeyer C, et al. Unraveling the gut microbiome of the long-lived naked mole-rat. Sci Rep 2017;7:9590.

31. Biagi E, Rampelli S, Turroni S, Quercia S, Candela M, Brigidi P. The gut microbiota of centenarians: signatures of longevity in the gut microbiota profile. Mech Ageing Dev 2017;165(Pt B): 180-4.

32. Bian G, Gloor GB, Gong A, Jia C, Zhang W, Hu J, et al. The gut microbiota of healthy aged chinese is similar to that of the healthy young. mSphere 2017;2:e00327-17.

33. Wang F, Yu T, Huang G, Cai D, Liang X, Su H, et al. Gut microbiota community and its assembly associated with age and diet in Chinese centenarians. J Microbiol Biotechnol 2015;25:1195204.

34. Odamaki T, Kato K, Sugahara H, Hashikura N, Takahashi S, Xiao JZ, et al. Age-related changes in gut microbiota composition from newborn to centenarian: a cross-sectional study. BMC Microbiol 2016;16:90.

35. Goodrich JK, Waters JL, Poole AC, Sutter JL, Koren O, Blekhman R, et al. Human genetics shape the gut microbiome. Cell 2014;159:789-99.

36. Wilson D, Jackson T, Sapey E, Lord JM. Frailty and sarcopenia: the potential role of an aged immune system. Ageing Res Rev 2017;36:1-10.

37. Casati M, Ferri E, Azzolino D, Cesari M, Arosio B. Gut microbiota and physical frailty through the mediation of sarcopenia. Exp Gerontol 2019;124:110639.

38. Grosicki GJ, Fielding RA, Lustgarten MS. Gut microbiota contribute to age-related changes in skeletal muscle size, composition, and function: biological basis for a gut-muscle axis. Calcif 
Tissue Int 2018; 102:433-42.

39. Siddharth J, Chakrabarti A, Pannerec A, Karaz S, Morin-Rivron $\mathrm{D}$, Masoodi M, et al. Aging and sarcopenia associate with specific interactions between gut microbes, serum biomarkers and host physiology in rats. Aging (Albany NY) 2017;9:1698-720.

40. Thevaranjan N, Puchta A, Schulz C, Naidoo A, Szamosi JC, Verschoor CP, et al. Age-associated microbial dysbiosis promotes intestinal permeability, systemic inflammation, and macrophage dysfunction. Cell Host Microbe 2018;23:570.

41. Buford TW, Carter CS, VanDerPol WJ, Chen D, Lefkowitz EJ, Eipers P, et al. Composition and richness of the serum microbiome differ by age and link to systemic inflammation. Geroscience 2018;40:257-68.

42. Lopez-Siles M, Duncan SH, Garcia-Gil LJ, Martinez-Medina M. Faecalibacterium prausnitzii: from microbiology to diagnostics and prognostics. ISME J 2017;11:841-52.

43. Lamoureux EV, Grandy SA, Langille MGI. Moderate exercise has limited but distinguishable effects on the mouse microbiome. mSystems 2017;2:e00006-17.

44.Bressa C, Bailen-Andrino M, Perez-Santiago J, Gonzalez-Soltero $\mathrm{R}$, Perez M, Montalvo-Lominchar MG, et al. Differences in gut microbiota profile between women with active lifestyle and sedentary women. PLoS One 2017;12:e0171352.

45. Clark A, Mach N. The crosstalk between the gut microbiota and mitochondria during exercise. Front Physiol 2017;8:319.

46. Kimura I, Inoue D, Hirano K, Tsujimoto G. The SCFA receptor GPR43 and energy metabolism. Front Endocrinol (Lausanne) 2014;5:85.

47. den Besten G, van Eunen K, Groen AK, Venema K, Reijngoud DJ, Bakker BM. The role of short-chain fatty acids in the interplay between diet, gut microbiota, and host energy metabolism. J Lipid Res 2013;54:2325-40.

48. Radak Z, Zhao Z, Koltai E, Ohno H, Atalay M. Oxygen consumption and usage during physical exercise: the balance between oxidative stress and ROS-dependent adaptive signaling. Antioxid Redox Signal 2013;18:1208-46.

49. den Besten G, Gerding A, van Dijk TH, Ciapaite J, Bleeker A, van Eunen $\mathrm{K}$, et al. Protection against the metabolic syndrome by guar gum-derived short-chain fatty acids depends on peroxisome proliferator-activated receptor $\gamma$ and glucagon-like peptide-1. PLoS One 2015;10:e136364.

50. Munukka E, Rintala A, Toivonen R, Nylund M, Yang B, Takanen A, et al. Faecalibacterium prausnitzii treatment improves hepatic health and reduces adipose tissue inflammation in high-fat fed mice. ISME J 2017;11:1667-79.

51. Bergman H, Ferrucci L, Guralnik J, Hogan DB, Hummel S, Karunananthan S, et al. Frailty: an emerging research and clinical paradigm: issues and controversies. J Gerontol A Biol Sci Med Sci 2007;62:731-7.

52. Kelaiditi E, Cesari M, Canevelli M, van Kan GA, Ousset PJ, Gillette-Guyonnet $S$, et al. Cognitive frailty: rational and definition from an (I.A.N.A./I.A.G.G.) international consensus group. J Nutr Health Aging 2013; 17:726-34.

53. Buchman AS, Bennett DA. Cognitive frailty. J Nutr Health Aging 2013;17:738-9.

54. Bosboom JL, Stoffers D, Wolters ECh. Cognitive dysfunction and dementia in Parkinson's disease. J Neural Transm (Vienna) 2004;111:1303-15.

55. Welcome MO. Current perspectives and mechanisms of relationship between intestinal microbiota dysfunction and dementia: a review. Dement Geriatr Cogn Dis Extra 2018;8:360-81.

56. Ticinesi A, Tana C, Nouvenne A, Prati B, Lauretani F, Meschi T. Gut microbiota, cognitive frailty and dementia in older individuals: a systematic review. Clin Interv Aging 2018;13:1497-511.

57. Sanguinetti E, Collado MC, Marrachelli VG, Monleon D, Selma-Royo M, Pardo-Tendero MM, et al. Microbiome-metabolome signatures in mice genetically prone to develop dementia, fed a normal or fatty diet. Sci Rep 2018;8:4907.

58. Xu R, Wang Q. Towards understanding brain-gut-microbiome connections in Alzheimer's disease. BMC Syst Biol 2016;10 Suppl 3:63.

59. Zhao Y, Jaber V, Lukiw WJ. Secretory Products of the human GI tract microbiome and their potential impact on Alzheimer's disease $(\mathrm{AD})$ : detection of lipopolysaccharide (LPS) in $\mathrm{AD}$ hippocampus. Front Cell Infect Microbiol 2017;7:318.

60. Araos R, Andreatos N, Ugalde J, Mitchell S, Mylonakis E, D’Agata EM. Fecal microbiome among nursing home residents with advanced dementia and clostridium difficile. Dig Dis Sci 2018; 63:1525-31.

61. Paley EL, Merkulova-Rainon T, Faynboym A, Shestopalov VI, Aksenoff I. Geographical distribution and diversity of gut microbial NADH:Ubiquinone oxidoreductase sequence associated with Alzheimer's disease. J Alzheimers Dis 2018;61:1531-40.

62. Alkasir R, Li J, Li X, Jin M, Zhu B. Human gut microbiota: the links with dementia development. Protein Cell 2017;8:90-102.

63. Degn SE, Jensen L, Olszowski T, Jensenius JC, Thiel S. Co-complexes of MASP-1 and MASP-2 associated with the soluble pattern-recognition molecules drive lectin pathway activation in a manner inhibitable by MAp44. J Immunol 2013;191:1334-45.

64. Friedland RP, Chapman MR. The role of microbial amyloid in neurodegeneration. PLoS Pathog 2017;13:e1006654.

65. Chen WW, Zhang X, Huang WJ. Role of neuroinflammation in neurodegenerative diseases (Review). Mol Med Rep 2016; 13:3391-6. 
66. Cattaneo A, Cattane N, Galluzzi S, Provasi S, Lopizzo N, Festari $\mathrm{C}$, et al. Association of brain amyloidosis with pro-inflammatory gut bacterial taxa and peripheral inflammation markers in cognitively impaired elderly. Neurobiol Aging 2017;49:60-8.

67. Qian Y, Yang X, Xu S, Wu C, Song Y, Qin N, et al. Alteration of the fecal microbiota in Chinese patients with Parkinson's disease. Brain Behav Immun 2018;70:194-202.

68. Delzenne NM, Cani PD, Daubioul C, Neyrinck AM. Impact of inulin and oligofructose on gastrointestinal peptides. Br J Nutr 2005;93 Suppl 1:S157-61.

69. Karaki S, Mitsui R, Hayashi H, Kato I, Sugiya H, Iwanaga T, et al. Short-chain fatty acid receptor, GPR43, is expressed by enteroendocrine cells and mucosal mast cells in rat intestine. Cell Tissue Res 2006;324:353-60.

70. Wei Y, Melas PA, Wegener G, Mathe AA, Lavebratt C. Antidepressant-like effect of sodium butyrate is associated with an increase in TET1 and in 5-hydroxymethylation levels in the Bdnf gene. Int J Neuropsychopharmacol 2014;18:pyu032.

71. Stilling RM, Ryan FJ, Hoban AE, Shanahan F, Clarke G, Claesson MJ, et al. Microbes \& neurodevelopment: absence of microbiota during early life increases activity-related transcriptional pathways in the amygdala. Brain Behav Immun 2015;50:209-20.

72. Desbonnet L, Clarke G, Traplin A, O’Sullivan O, Crispie F, Moloney RD, et al. Gut microbiota depletion from early adolescence in mice: implications for brain and behaviour. Brain Behav Immun 2015;48:165-73.

73. Sun P, Zhou K, Wang S, Li P, Chen S, Lin G, et al. Involvement of $\mathrm{MAPK} / \mathrm{NF}-\kappa \mathrm{B}$ signaling in the activation of the cholinergic anti-inflammatory pathway in experimental colitis by chronic vagus nerve stimulation. PLoS One 2013;8:e69424

74. Sacknoff DM, Gleim GW, Stachenfeld N, Coplan NL. Effect of athletic training on heart rate variability. Am Heart J 1994; 127:1275-8.

75. Furlan R, Piazza S, Dell’Orto S, Gentile E, Cerutti S, Pagani M, et al. Early and late effects of exercise and athletic training on neural mechanisms controlling heart rate. Cardiovasc Res 1993;27: 482-8.
76. Owen N, Sparling PB, Healy GN, Dunstan DW, Matthews CE. Sedentary behavior: emerging evidence for a new health risk. Mayo Clin Proc 2010;85:1138-41.

77. Gonzalez-Freire M, de Cabo R, Studenski SA, Ferrucci L. The neuromuscular junction: aging at the crossroad between nerves and muscle. Front Aging Neurosci 2014;6:208.

78. Silverman MN, Deuster PA. Biological mechanisms underlying the role of physical fitness in health and resilience. Interface Focus 2014;4:20140040.

79. Cerda B, Perez M, Perez-Santiago JD, Tornero-Aguilera JF, Gonzalez-Soltero R, Larrosa M. Gut microbiota modification: another piece in the puzzle of the benefits of physical exercise in health? Front Physiol 2016;7:51.

80. Matsumoto M, Inoue R, Tsukahara T, Ushida K, Chiji H, Matsubara N, et al. Voluntary running exercise alters microbiota composition and increases n-butyrate concentration in the rat cecum. Biosci Biotechnol Biochem 2008;72:572-6.

81. Allen JM, Berg Miller ME, Pence BD, Whitlock K, Nehra V, Gaskins $\mathrm{HR}$, et al. Voluntary and forced exercise differentially alters the gut microbiome in C57BL/6J mice. J Appl Physiol (1985) 2015;118:1059-66.

82. Hsu YJ, Chiu CC, Li YP, Huang WC, Huang YT, Huang CC, et al. Effect of intestinal microbiota on exercise performance in mice. J Strength Cond Res 2015;29:552-8.

83. Denou E, Marcinko K, Surette MG, Steinberg GR, Schertzer JD. High-intensity exercise training increases the diversity and metabolic capacity of the mouse distal gut microbiota during diet-induced obesity. Am J Physiol Endocrinol Metab 2016;310:E98293.

84. Mika A, Van Treuren W, Gonzalez A, Herrera JJ, Knight R, Fleshner M. Exercise is more effective at altering gut microbial composition and producing stable changes in lean mass in juvenile versus adult male F344 rats. PLoS One 2015;10:e0125889.

85. Petriz BA, Castro AP, Almeida JA, Gomes CP, Fernandes GR, Kruger RH, et al. Exercise induction of gut microbiota modifications in obese, non-obese and hypertensive rats. BMC Genomics 2014;15:511. 